\title{
Investigating Variations in Gameplay: Cognitive Implications
}

\author{
Kamran Sedig, Robert Haworth, and Michael Corridore
}

Middlesex College, Western University, London, ON, Canada N6A 5B7

Correspondence should be addressed to Kamran Sedig; sedig@uwo.ca

Received 30 May 2014; Revised 25 November 2014; Accepted 22 December 2014

Academic Editor: Alexander Pasko

Copyright (C) 2015 Kamran Sedig et al. This is an open access article distributed under the Creative Commons Attribution License, which permits unrestricted use, distribution, and reproduction in any medium, provided the original work is properly cited.

\begin{abstract}
There is increasing interest in creating computer games for learning, problem solving, and other high-level cognitive activities. When investigating whether gameplay is conducive to such activities, gameplay is often studied as a whole. As a result, cognitive implications can be linked to the game but not to its structural elements. Given that gameplay arises from interaction between the player and the game, it is the structural components of interaction that should be investigated to better understand the design of gameplay. Furthermore, minor variations in the components of interaction can have significant cognitive implications. However, such variation has not been studied yet. Thus, to gain a better understanding of how we can study the effect of interaction on the cognitive aspect of gameplay, we conducted an exploratory investigation of two computer games. These games were isomorphic at a deep level and only had one minor difference in the structure of their interaction. Volunteers played these games and discussed the cognitive processes that emerged. In one game, they primarily engaged in planning, but in the other game they primarily engaged in visualizing. This paper discusses the results of our investigation as well as its implications for the design of computer games.
\end{abstract}

\section{Introduction}

Computer games have now become popular and ubiquitous in our society, especially as the proliferation of games on social media and cell phones increases. At the same time, interest in using computer games in nonentertainment contexts has been increasing (e.g., [1-3]). Games within most of these contexts are more effective when they engage the player in deep and meaningful cognition, for example, reflective learning of mathematics, solving complex engineering problems, and planning a sequence of actions in order to accomplish some goal. Therefore, we need to design these games with conscious attention given to their cognitive gameplay. Generally speaking, the term gameplay refers to the experience which emerges from the interaction between the player and the game $[4,5]$. Cognitive gameplay refers to the cognitive processes that emerge from the player interacting with the game. In other words, cognitive gameplay is the cognitive component of the experience of playing a game. The quality of cognitive gameplay is the primary factor affecting the ability of a game to engage the player in cognitive tasks and activities [6]. For example, if we wanted to create a computer game for mathematics education, the game should engage the player in the cognitive activity of learning specific mathematical concepts. Hence, it would be insufficient to simply embed these concepts into the game; we would also need to carefully design the cognitive gameplay so that the game is conducive to deep and conscious reflection of the embedded mathematical concepts (see $[6,7]$ ).

Design of cognitive gameplay is a second-order design problem $[5,8]$. We cannot directly design gameplay, since it emerges from the interaction that occurs between the player and the game. Instead, we must design the interaction afforded by the game, as it is from this interaction that gameplay emerges. In other words, we can control the resulting gameplay by carefully designing the interaction. Similarly, to design cognitive gameplay we need to design the interaction so that it facilitates, promotes, and supports the desired cognitive engagement [9]. For example, if we wanted to design a game for learning mathematics, and thus wanted cognitive gameplay that is conducive to learning, then we need to design the game's interaction in such a way that it enables and promotes deep and conscious reflection upon the embedded mathematical concepts.

Currently, there are no frameworks or methods for the systematic design of cognitive gameplay. Many of the existing 
frameworks and methods for designing gameplay tend to focus on the noncognitive components of gameplay, such as enjoyment, immersion, or challenge (e.g., [10-13]). Cognitive gameplay can influence noncognitive components, such as enjoyment, but a framework that focuses on these other components does not inform us about how to engage the player in specific cognitive tasks or activities. Other frameworks relate specific structural features of games to learning (e.g., $[14,15])$. While these frameworks are more useful for the design of cognitive gameplay, the structural features on which they focus are quite broad (e.g., narratives, evaluation, and personalization); usually they are not directed toward interaction design. In addition, these frameworks focus on the specific cognitive activity of learning and hence may not generalize well to other high-level cognitive activities, for example, decision making, planning, or problem solving.

Sedig and colleagues [16] have developed a framework that informs the design of interactivity (i.e., the quality of interaction) at a microlevel for visual tools. Although the current context of this framework is visual tools, aspects of it may be applicable to the design of computer games. Specifically, this framework could inform the design of microlevel interactivity in a computer game and thus inform the design of cognitive gameplay to some extent (see [9]). However, to further understand the systematic design of cognitive gameplay, we need to investigate the relationship between microlevel interactivity and cognitive gameplay. In a more general sense, we need a method for studying the effects of structural elements of computer games on cognitive gameplay.

Typical studies of cognitive gameplay investigate the relationship between the game as a whole and some internal change in the player (see [11, 17]). For example, Quiroga and colleagues [18] conducted a study to determine whether playing Big Brain Academy for multiple trials would influence general intelligence test scores. The results of their study indicated that some test scores were influenced and, thus, the cognitive gameplay of Big Brain Academy involves general intelligence. Although these results are important and useful, they do not help us determine what component(s) of Big Brain Academy were responsible for this effect. For instance, we cannot conclude from this study whether the interaction afforded by the game influenced the results of the general intelligence test; other components of the game may be responsible, such as the game's content, the manner in which this content is represented, and the game's goals. Without a method of identifying and isolating components of a game, it is very difficult to determine the source of any cognitive effect. Thus, while this study is still valuable, it does not further our understanding of how to design a computer game's essential interactions in order to create some desired cognitive gameplay. Although other studies that investigate the effect of particular components of a computer game on cognitive gameplay (e.g., [19]) are more useful, they are still insufficient. Their emphasis tends to be on a particular cognitive activity (e.g., knowledge acquisition), and, as previously mentioned, the results may not be generalizable to other high-level cognitive activities. The lack of generalizability is primarily due to the methods used for designing the tested computer games.

To study the design of cognitive gameplay, we need a systematic process for design that is (1) general in terms of the possible cognitive activities that can result and (2) capable of isolating specific game components for further study. The process for designing computer games presented in [20] may be applicable for this purpose, but it has not been tested. Hence, we investigated whether this process would assist in studying the design of cognitive gameplay. We conducted a simple investigation of the cognitive gameplay of two computer games. These games were designed using the process described in [20] and were designed such that structural differences could be isolated and controlled. To test whether it was possible to isolate only one structural difference, we designed these games so that the only structural difference between them was one element of interaction. When these games were played by our volunteers, differences in cognitive gameplay were observed. These differences were based on self-reported descriptions of the volunteers' experiences and their own observations of the differences between the games. The difference in cognitive gameplay could be associated with the structural differences in interaction between the two games. In other words, we found some promising evidence that (1) careful design of interaction can affect cognitive gameplay, (2) these effects can be studied in a systematic and controlled manner, and (3) the design process mentioned in [20] could be of benefit for such studies. However, this is still preliminary research.

In this paper, then, we will present the results of this investigation and some of the theoretical considerations surrounding the investigation. In Section 2 below, some of the design concepts of computer games will be discussed as well as the design process used for the two games. Following this, in Section 3, the two games and the investigation procedure will be explained. Then, in Section 4, the results of the investigation will be presented, followed by a discussion in Section 5 . Some conclusions about how this investigation furthers our understanding of the design of cognitive gameplay will be presented at the end, in Section 6.

\section{Background}

In this section we will discuss some of the background concepts and terminology before we present our investigation.

2.1. Computer Games. Many terms have been used throughout the literature to describe the kind of games that we talk about in this paper, such as video game, digital game, or electronic game. We will simply adopt the term computer game, though the other terms can be considered as equally applicable. By computer game we mean a game that is implemented on some form of computational platform. The physical hardware used is unimportant, as the concepts in this paper equally apply to a game that functions on a personal computer, mobile device, tablet computer, or game console. However, using computational technology as the means through which interaction occurs gives computer games a greater degree of interactive potential. This is due to the 
interaction that can be designed for computer games as well as the removal of physical limitations inherent in noncomputer games (e.g., card games, board games, and physical sports). As a result of this interactive potential, computer games also have a greater degree of cognitive potential and higher potential for more variety and depth of cognitive gameplay.

2.2. Cognition. Over the past few decades, researchers in cognitive science have increasingly promoted the idea that human cognition is fundamentally influenced by the surrounding environment (e.g., [21-24]). Although there are cognitive processes that operate within a human, such internal processes do not simply operate on some decontextualized input. Artifacts external to a human can cause the operation of internal processes to be augmented, constrained, or offloaded. In other words, one can conceptualize the operation of cognitive processes as though they were distributed across multiple objects in an environment $[25,26]$.

A more comprehensive model for cognition, in which external artifacts are taken into account, is one where cognitive processes occur within a joint human-artifact cognitive system. This model allows us to analyze the whole cognitive system rather than merely focus on internal cognitive processes in isolation of the environment. The cognitive system most relevant to this paper is the one composed of a player and a computer game. The player is a subsystem, composed of internal cognitive processes, expectations towards games, skill with certain games, and so on. The computer game is also a subsystem, composed of various User Interface (UI) elements which the player perceives and with which she interacts. By analyzing the whole player-game cognitive system we can identify the way in which cognitive processes are distributed across the player and the computer game (for an example of this in a context outside of computer games, see [27]).

For example, Kirsh and Maglio [28] studied people playing the computer game Tetris and found that certain cognitive processes were extended into the game. In Tetris, the player arranges differently shaped blocks in a play space so as to create horizontal rows. To do this, she needs to quickly determine the location and orientation of each block and arrange it accordingly. Arranging a block involves a combination of two operations: rotating and translating. To determine the best location and orientation of a block, the player could internally visualize the result of possible operations on it. However, Kirsh and Maglio found that their participants would often operate on blocks in the game and use the result of those operations to determine the best location and orientation of blocks. Instead of internally visualizing the result of an operation the participants simply performed the operation, saw the result, and used this information to help them arrange the corresponding block. They would do so despite having limited time to act on any block and despite the potential risk of having the block end up in a location or orientation that was detrimental to their progress. Performing such operations on a block is an example of a cognitive process being distributed within the player-game cognitive system: the player offloaded her cognitive process of visualizing onto the game when she operated on blocks in the game instead of internally visualizing the same operation.

2.3. Interaction. The distribution of cognitive processes is enabled by the relationships that exist between elements in the cognitive system. In the case of the player-game cognitive system, the most influential relationship is the interaction that occurs between the player and the computer game. A single interaction has two components: an action that the player performs on one or more elements of the UI and a reaction from the game. By reaction is meant some perceivable change of the UI, such as an element of the UI changing its position or new UI elements being created. In playing the game, the player performs an action, the game responds with a reaction, the player perceives this reaction, and then the player performs a new action. As this actionreaction cycle repeats, the player and the game become coupled together into a two-way mutual dialogue (see $[29,30]$ for elaboration, even though they are not in the context of computer games). Cognition emerges from this dialogue, in that the interaction engages and mediates cognitive processes and facilitates their distribution [16].

2.4. Interactivity. The term interactivity refers to the quality of interaction, and therefore it means the quality of the cognitive coupling between the player and the computer game [9]. We can conceptualize an interaction in a computer game as a composite of a set of elements that collectively determine its structure. In the context of the design of visual tools, Sedig and colleagues [16] have identified twelve such elements, which could also apply to the design of computer games; some of these elements include activation, context, flow, and focus. These elements have various operational forms that affect the quality of the cognitive coupling between the player and the game. Thus, designing interactivity of a game involves determining how the elements of its interactions should be operationalized.

For example, one of the structural elements of interaction is focus. Focus can be operationalized as direct or indirect. In a game with direct focus the player acts on a target UI object, while in a game with indirect focus the player acts on an intermediary UI object to influence a target UI object. In a study that explored designing computer games for learning mathematical concepts [19], two computer games were developed that differed in the way in which focus was operationalized (though the authors of the study do not use the term focus). The results of this study indicated that there were differences in cognitive gameplay; specifically, the game with indirect focus led to deeper and more effortful learning of the embedded mathematical concepts than the game with direct focus. In other words, operationalizing this structural element of interaction in one way resulted in cognitive gameplay that was characterized by deep and mindful learning, while operationalizing it in a different way resulted in cognitive gameplay that promoted shallow and habituated learning. This study is also an example of how we could investigate the effect of structural differences of interaction on cognitive gameplay. However, in [19] there was no discussion of a systematic and generalized method for the design or 
investigation of their games. As previously mentioned, they also focused entirely on learning (specifically acquisition of mathematical knowledge) and made no indication that their study could be generalized to other cognitive activities.

2.5. Cognitive Gameplay. Roughly speaking, the term gameplay refers to the subjective experience of the player while playing a game [5]. However, it can also refer to the features of a game that affect the kind of experience that the player may have (e.g., $[8,31])$. In this paper though, we will use the definition provided by Ermi and Mäyrä, who say that gameplay "is not a property or a direct cause of certain elements of a game but something that emerges in a unique interaction process between the game and the player." (page 2, [4]) Although the experience that emerges is still subjective, interaction is what facilitates and mediates its emergence.

Cognitive gameplay is a subset of gameplay and can be defined as the cognitive processes that emerge through the mutual dialogue between the player and the game [9]. In other words, it refers only to the cognitive aspect of gameplay. Other aspects of gameplay can be influenced by cognitive gameplay and vice versa, but the term cognitive gameplay does not refer to these. For example, the pleasure gained from aesthetic features of a game, the degree of visual immersion or engagement, and the perception of challenge are all aspects of gameplay but are not part of what we mean by cognitive gameplay. The player mindfully and deliberately determining the best position for some object, planning a path through a navigation space, devising a strategy to solve a puzzle, deciding which options to take, taking into account a concept to overcome an obstacle, creating a sequence of actions to create a structure, or trying to remember a previously failed attempt so as to avoid it are all examples of experiences captured by the term cognitive gameplay.

Since both gameplay and cognition emerge from interaction between the player and the computer game, we can design cognitive gameplay by designing the interaction of the computer game. Specifically, it is interactivity design that is most relevant for designing cognitive gameplay. This is because interactivity determines the quality of the cognitive coupling between the player and the game, and hence interactivity determines the quality of the resulting cognitive gameplay $[9,16]$. Likewise, we can gain a better understanding of how to systematically design cognitive gameplay through investigating the effect of a computer game's interactivity on its resulting cognitive gameplay.

2.6. Investigating Cognitive Gameplay. In order to investigate cognitive gameplay, it is first necessary to identify the structural component(s) of the computer game that will be explored. Simple computer games are often best for this, since they have few structural components. For example, the computer game Tetris has only two actions (rotate a block and translate a block), a handful of reactions, and one game object (the Tetris block in its various forms). However, to determine whether a component influences cognitive gameplay, we need to vary that component and compare games which have those variations. Comparing the gameplay of one computer game with the gameplay of a different computer game can be problematic, as both games require deep structural similarity before differences between them can be comprehensively identified. In other words, the two games need to be isomorphic at a deep level. When two games are isomorphic it means that they are structurally identical at a certain level of abstraction (e.g., see [32]). The level of abstraction can vary, such that two games may be isomorphic at one level but not at a different level.

For example, consider two games that are variations of Tetris. In one game, the blocks are multicolored and glow when translated or rotated. In the other game, the whole display is black-and-white. These two games are different at the surface level of abstraction, or the presentation level of information, but they are isomorphic at deeper levels since they share the same rules and interactions. As another example, consider two other variations of Tetris in which there is one difference between them: in one game only the next block that will appear is shown, while in the other game the next four blocks that will appear are shown. This change is at the level of rules and hence is deeper than the presentation level. Thus, these two games are not isomorphic at the surface level or at the level of rules. However, they are still isomorphic at a more abstract level where both games share the abstract rule "present the next set of blocks." In one game this abstract rule is specified as "show the next block" and in the other game the rule is specified as "show the next four blocks."

A design process was developed and proposed by Sedig and Haworth [20] in which multiple games can be designed and the structural differences between these games can be identified and controlled. Through this process, a series of games could be produced that are isomorphic at a very abstract level: they share the same source idea. In addition, the games can maintain isomorphism at other levels of abstraction, depending on how they are designed. The process involves five stages: (1) choosing a cognitive toy as a source of inspiration, (2) extracting some general patterns from the toy, (3) choosing one of the general patterns and using it to design a set of abstract rules, (4) using the abstract rules to create a set of concrete rules, and (5) using the concrete rules to implement a full computer game. Each stage after the first can be performed multiple times, branching out into separate paths for creating a computer game. For instance, one cognitive toy could be chosen, from which two sets of general patterns could be extracted. Three sets of abstract rules could be generated from each general pattern, giving a total of six sets of rules, and eventually resulting in at least six different computer games that are isomorphic to varying degrees. However, there was no mention in [20] of this process being used to empirically investigate the effect of structural differences of interaction on cognitive gameplay, although such a possibility was implied.

An example of using this process to create games is shown in Figure 1. A cognitive toy is chosen and four different computer games are produced at the end of the process. All four games are isomorphic at a very deep level, since they are all based on the same cognitive toy. The games are also isomorphic at the level of abstract rules, since they share the same set of abstract rules. The two games in the middle are isomorphic at the level of concrete rules, since they share the 


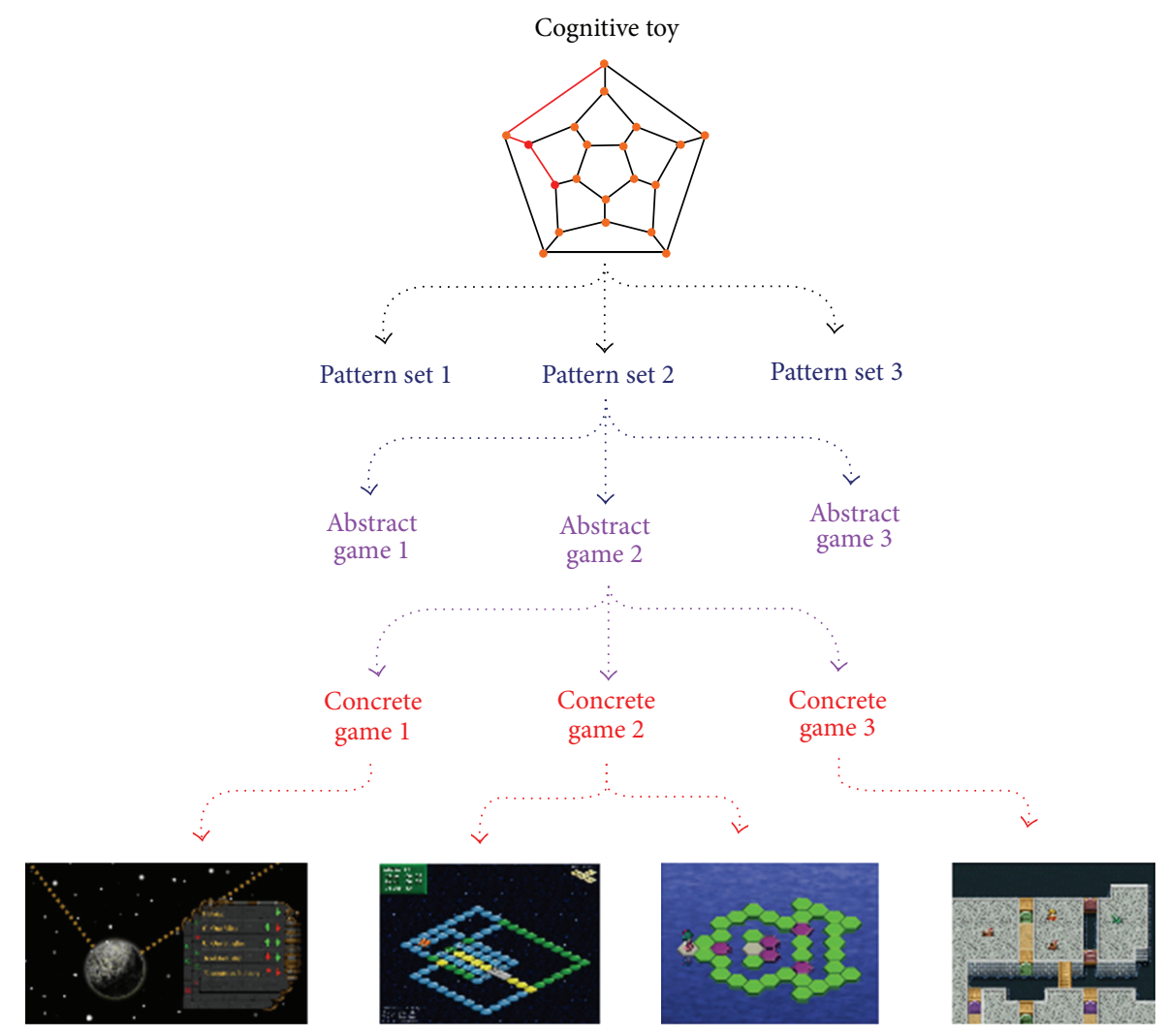

FIgURE 1: An example of the design process discussed in [20] being used to create four isomorphic computer games.

same set of concrete rules. The four games are different at the presentation level, and the first and fourth games are different at the level of concrete rules. The structural changes between the first and second games are much greater than the changes between the second and third games, given the difference in degree of isomorphism between those three games.

The stage in the process from which two games diverge determines the depth and degree of isomorphism. Since the designer determines when divergence occurs in the process, and the design that is created at each stage, he therefore controls the degree and kind of difference that occurs between the resulting games. In other words, by using this process we can create multiple games that differ in terms of exactly the structural component(s) that we want to investigate.

\section{Methodology}

In this section we will briefly discuss the two isomorphic computer games that we developed and the procedure for our investigation.

3.1. Computer Games. For our investigation of the effect of structural variations of interaction on cognitive gameplay, two isomorphic computer games were developed: Fixed Play Space (Game F) and Rotating Play Space (Game R). Both are $2 \mathrm{D}$ maze-like puzzle games that were developed by our research team using the design process and theoretical concepts described in Section 2.

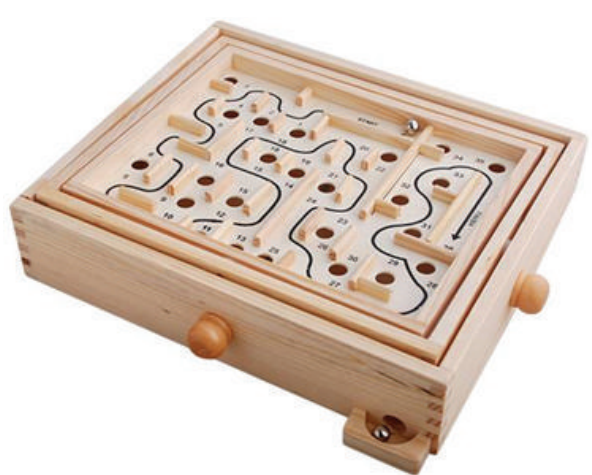

FIGURE 2: A photo of a classic Labyrinth, which was the inspiration for the two computer games that we developed.

The cognitive toy Labyrinth (see Figure 2) was chosen in the first stage of the design process. In this toy, the player turns dials to tilt the board and cause a marble to roll in a certain direction. The objective is to move the marble from a start position to an end position such that it does not fall into one of the many holes in the board. From this toy, we designed a simple abstract game, in which the player must navigate an orb from a start position to a goal position while avoiding various obstacles. Some obstacles stop the orb from moving while others force the player to restart. The orb moves in a random direction each time it collides with an obstacle. In addition, the player can arrange a limited number of 


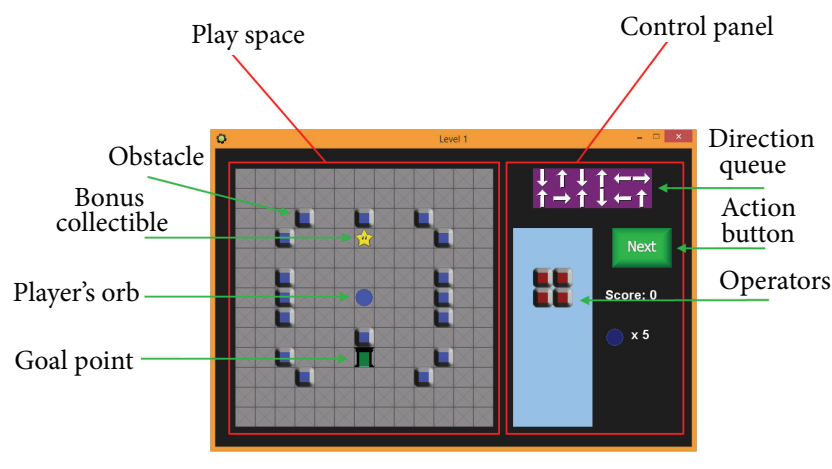

Figure 3: Screenshot of Game F with its UI components labeled.

operators. These operators cause the orb to move in a specific direction or to stop moving. Thus, the player cannot directly move the orb but she can influence its direction by using various operators.

From this abstract game we derived two concrete rule sets and then implemented those rules as two computer games. Both games are divided into eight levels. Each level has a start point, a goal point, a specific arrangement of obstacles and bonus collectibles, and specific operators available to the player. The levels are intended to be progressively more difficult, based on the obstacles and the number and kind of operators available. The random directions of the orb were instantiated as a direction queue. This queue shows a sequence of twelve directions, and the player can click an action button to cause the orb to move in a way related to the first entry in the direction queue. The first entry is then removed and a new random one is added to the end, effectively making the queue infinite in length.

The UI is divided into two parts: the play space and the control panel (see Figure 3). The play space contains the orb and the level-specific arrangement of elements (the goal, bonus collectibles, and obstacles). This is also the space in which the player places and arranges the operators available for the current level. The control panel contains the direction queue, the available operators, the action button, and the player's current score and remaining lives.

In addition to clicking the action button, the player can arrange the given operators. This is done by clicking on an operator in the control panel and dragging it onto the play space. The player can move an operator after placing it, but she cannot move operators while the orb is in motion nor can she hold an operator somewhere while the orb is moving. In other words, operators must be placed and then left alone while the orb moves. An operator can be placed anywhere on the play space except on top of another operator or within one tile of the orb. Hence, the player cannot place any operator directly beside the orb. There are two types of operators in the game: walls and arrows. When the orb collides with the wall operator, the orb stops moving. Wall operators give the player more control over the ending position of the orb. When the orb collides with the arrow operator, it moves again in the direction of the arrow. For example, if an orb was moving to the right and collided with an "up" arrow, the orb would then begin moving upward and would continue until it collided with something else. Arrow operators give the player more control over the direction of the orb's movement. The player was not given the same number or type of operators in each level; in some levels the player may have walls and arrows, while in others she may have only arrows or only walls.

Bonus objects are placed throughout each level. One type of bonus object awards the player 50 points when the orb collides with it. The player cannot receive points in any other way. The other type of bonus object awards the player an extra life when the orb collides with it. About halfway through the game, black hole objects were also placed on the level. When the orb collides with a black hole, the player loses a life and the level restarts. When all the player's lives are lost, the game ends in failure. Colliding with a black hole is the only way in which the player can lose lives and thus is the only way in which she can lose the game. If the player reached the goal point of the final level, she wins the game and is shown her final score. We did not attempt to provide any other scoring mechanism, such as points for operators used or time spent per level, as these might become additional factors influencing the behavior of players.

Slightly different graphics were used in each game, which means that the two games differed at the presentation level. The games also had different directions in the direction queue and responses to the action button being clicked. In other words, the games differ in the reaction component of interaction. This is the structural difference that we explored. Other than this one structural difference, and the presentation differences, the two games are identical.

3.1.1. Fixed Play Space (Game F). In Game F, the direction queue contains a series of cardinal directions: up, down, left, and right. When the action button is clicked, the orb begins moving in a straight line in the direction of the first entry in the queue and stops when it collides with a wall or the edge of the play space. There is an animation of the orb as it moves, so that the player can see it move from its start to end position. Only the orb moves; all other objects in the play space remain fixed in place. See Figure 4 for an example of this movement. If the orb collides with an arrow operator, it moves in the same fashion (i.e., gradually until it collides with another operator). The goal point is presented as a door, the bonus collectible that gives score is presented as a star, and the bonus collectible that gives an extra life is presented as a circle that looks similar to the player's orb. The wall operators have the same appearance as existing walls except for a difference in color.

3.1.2. Rotating Play Space (Game R). In Game R, the direction queue contains a series of rotation directions: 90-degree clockwise turn, 90-degree counter-clockwise turn, and a 180degree turn. When the action button is clicked, the play space and all elements within it, including the orb, rotate according to the first entry of the direction queue. This rotation is animated (i.e., the player can see the objects move). After the rotation is finished, the orb moves down towards the bottom of the play space. This movement is similar to the orb's movement in Game F (i.e., gradual, animated, and stopping 


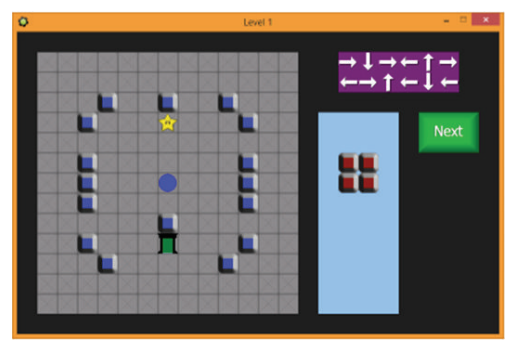

(a)

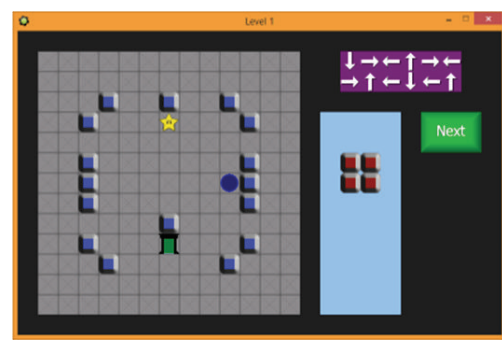

(b)

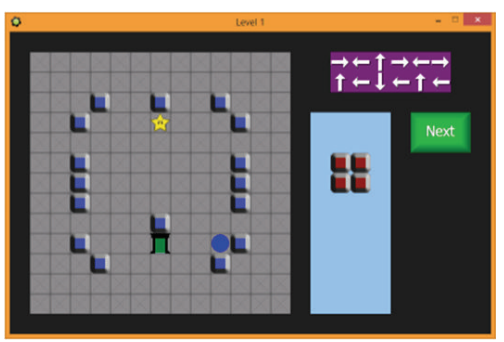

(c)

FIGURE 4: Screenshots from Game F that show the ending position of the orb after the player clicks the action button. From left to right: the starting position, after one movement right, and after one movement down.

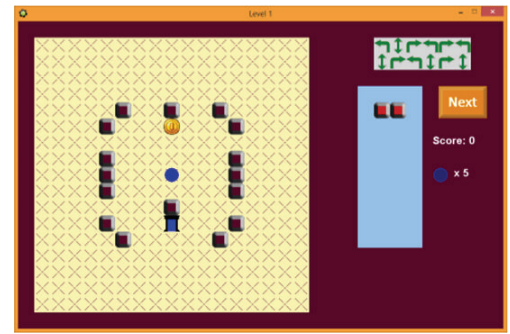

(a)

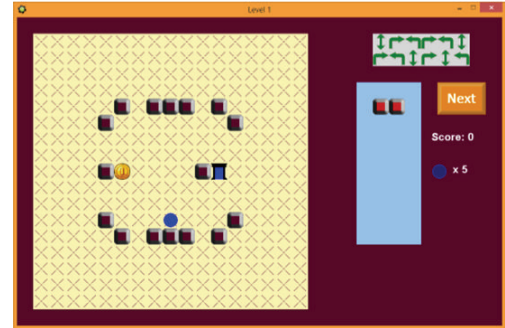

(b)

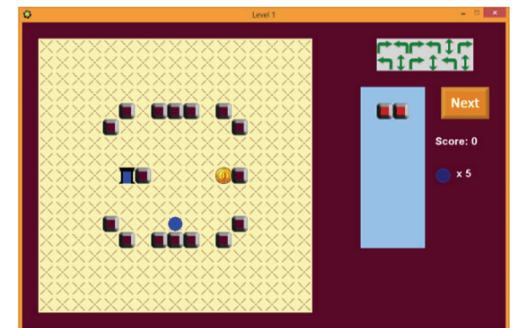

(c)

FIGURE 5: Screenshots from Game R that show the orb moving. From left to right: the starting position, after a 90-degree left rotation, and after a 180-degree rotation.

upon colliding with another object or the edge of the play space). See Figure 5 for an example of the rotation. The orb does not collide with anything during the rotation, only afterwards when it is moving down toward the bottom of the play space. The game objects look the same as in Game F except minor color differences in the background, UI, and walls, and the bonus collectible that gives score is presented as a coin instead of a star.

3.2. Investigation Procedure. We recruited eight volunteers from our university through word-of-mouth to participate in our exploratory investigation $(N=8)$. These volunteers are henceforth referred to as players. None of them had previously played the games that we had created. These players were randomly divided into two groups: Group 1 and Group 2. The procedure for our investigation was divided into four parts: (1) players filled out a demographics survey on paper; (2) they played one of the two games for about 30 minutes; (3) they filled out a design questionnaire on paper; (4) they played the other game for about 30 minutes. The whole procedure lasted about 90 minutes per person. People in Group 1 played Game F and then Game R, while those in Group 2 did the reverse.

We collected 4 types of data to analyze cognitive gameplay: (1) video-recordings of the game screen, which captured in-game interaction of the players; (2) audio-recordings, which captured their verbal comments; (3) two paper surveys, which captured specific opinions about the game and the experienced gameplay; and (4) direct observations obtained from watching the players interact with the game. The demographics survey was intended to gather past game-playing behavior and preferences to provide context for their comments. The design questionnaire was intended to gather their opinions about the game. For example, they were asked: whether they liked the game and why, whether they had any difficulties playing the game, the amount and nature of challenge the game provided, the kind of cognitive tasks in which they engaged, and the mental effort involved in these tasks. The design questionnaire only applied to the first game that they played.

For each game, players were given a one-minute summary of how to play the game. We answered any questions they had about how to play either game. While playing the first game, we asked them some questions to help elaborate or explain their behavior and to encourage them to vocalize their thoughts and the operation of their internal cognitive processes. When playing the second game, they were asked these same questions as well as a set of specific interview questions to encourage them to compare the two games. For example: do you like this game more than the previous one and why? Which game required more mental effort? What kind of mental effort was involved? Which game would you prefer to play again and why?

We were primarily interested in two types of observations. First, we wanted to explore how players experienced the game in cognitive terms. Specifically, we wanted to know what players were thinking about, what they were mentally emphasizing, and why they were engaged in this behavior. If players found it difficult to verbally express these experiences, or felt as though they were not thinking strongly about 
anything, then we could interpret this to mean they were not aware of their thoughts; in other words, the cognitive gameplay was more automatic and unconscious than reflective and conscious. Although we might want a game in which players think more deeply or are more aware of their thinking, we did not want to measure that in this study nor did we want to conclude which game was better. Our interest was to explore any possible differences in cognitive gameplay between playing these two games. Second, we wanted to explore differences in perceived enjoyment. We were interested in players indicating whether they enjoyed one game over the other, if they experienced any frustration, and explaining their thoughts on the source of this enjoyment or frustration. While this whole investigation could be conducted using quantitative means (see Section 5.4), collecting qualitative data was deemed more appropriate. Furthermore, the extremely low sample size rendered quantitative analysis irrelevant. Within the standard expectations of quantitative studies (e.g., $\alpha=0.05$; effect size $=0.1$; power $=0.8$ ) the statistical power of our sample is roughly 0.05 , far below what is needed to make any generalizable claims (see [33]). As such, quantitative analysis was deemed unnecessary and only qualitative results will be presented.

\section{Results}

This section is divided into three subsections. First, we provide a brief summary of some data gathered from the demographics surveys and scoring, to obtain a clearer picture of the players. The remaining two subsections contain the qualitative results for the two games, one section per game. Each subsection begins with a summary of the results for the corresponding game and continues with more detailed results. These results include a combination of comments from the players, their responses on the paper surveys, and observations made by the researchers. Where relevant, we mention whether the player was in Group 1 or Group 2. Players are referred to as $\mathrm{P}<\#>$ (e.g., P1, P5) when they are quoted in the results. The quotes are verbatim, minus the removal of filler words (e.g., "uhh," "umm," "like," and "you know”). We have reported and emphasized the qualitative results over the quantitative results, even though some quantitative data is reported.

4.1. Player Demographics and Scoring. In this subsection, we present some of the demographic data gathered about the players (see Table 1). This data is sorted into the two groups to help clarify the context of detailed results given in Sections 4.2 and 4.3. Statistical analysis of this data has not been conducted due to the small sample size. However, it is interesting to note that three of the players self-identified as nongamers and none of the players identified puzzle games as a type of game with which they had experience playing.

In Tables 2 and 3 we present some scoring results. The scores that players obtained could be interpreted as representing their level of motivation for obtaining optional content in the games, since score was not awarded for merely completing a level. Obtaining a high score could mean that the player was willing and interested in additional challenge; obtaining a low score could mean that the player was unwilling or unable to go after the bonus objectives in the game. The maximum score in both games was 1100. For added context with interpreting this data, the highest level reached in the game is also reported. Each game had 8 levels, and Tables 2 and 3 report the level of the game at which the experiment ended. An entry of "end" means that the player completed the game. Total play time for each player per game was roughly the same: 30 minutes. Again, this data was not analyzed statistically due to low sample size. Relevant data for P8 was lost due to a recording error.

4.2. Results of Game F. Players in Group 1 played Game F first, while those in Group 2 played it second. In this game they reported that the main cognitive task in which they were engaged was planning, planning in Game $F$ required less mental effort than in Game R, Game F had a low level of difficulty, Game F's low level of difficulty affected how deeply they planned, and Game F was more enjoyable than Game R but considered trivial in comparison.

4.2.1. Main Cognitive Task: Planning. Planning a path seemed to be the primary cognitive task in which players engaged. For example, the following is a portion of $\mathrm{P} 2$ verbalizing his thoughts while working through one level:

"First of all, if I go up all the way to the upper
corner here it won't be good for me, because the
ball will go up and then it will go right and then it
will go up and... up and down and then up and
to the right. So, this is the level where I really need
to use at least one of these [wall operators]. Now, I
have four so I need to create a path based on them.
So, if I go up, these two are probably the best places
where I need to stop the ball, which means that
I have to put something-one of those blocks-in
either of those two cells. If not, I have to avoid this
[one location in the play space] because the next
move will go towards the black hole. So then if I
go to the right, I cannot stop here of course, or here
because the next move is up and then I will lose."

The fact that the players engaged in planning could also be seen in other ways. While reflecting on the game, P8 wrote in the design questionnaire that the game "required a decent amount of foresight for some of the levels, which if you did not do, may result in death or a very long time to beat it." While playing the game, players pointed to specific bonuses or locations in the play space which they were trying to reach and planned a path to it. P4 explained that, "my strategy is to get the highest amount of points. I am just looking at the next directions, and trying to predict the first direction." Later on, when creating a plan in a level with several objectives, P4 continued to elaborate his strategy: "Right now I do not care about these directions [the ones near the end of the queue], I just pick one goal-one star or one life-and match the first direction to where I want to go."

Players mainly engaged in one kind of behavior to externalize the path they were planning: they would trace 
TABLE 1: Summary of player demographics.

\begin{tabular}{lcccc}
\hline Age $^{1}$ & Gender & Gamer status $^{2}$ & Experience $^{3}$ & Experienced game genres $^{4}$ \\
\hline 28 & & & Group 1 & FPS, sports, strategy \\
29 & M & NG & E & Cell phone games, word games \\
22 & F & NG & SE & Adventure, sports \\
22 & M & C-A & SE & FPS, sports, strategy \\
\hline & M & A & VE & Adventure, mystery, sports \\
28 & & & Group 2 & MMORPG, strategy \\
26 & M & C & & SE \\
22 & M & H & X & Adventure, FPS, RPG, sports \\
23 & M & C-A & VE & Adventure, FPS, RPG \\
\hline
\end{tabular}

${ }^{1}$ Ages are in years.

2 "Do you consider yourself:" $\mathrm{NG}=$ nongamer, $\mathrm{C}=$ causal gamer, $\mathrm{C}-\mathrm{A}=$ between $\mathrm{C}$ and $\mathrm{A}, \mathrm{A}=$ average gamer, $\mathrm{A}-\mathrm{H}=$ between $\mathrm{A}$ and $\mathrm{H}$, and $\mathrm{H}=$ hardcore gamer.

“"In general, how experienced are you with playing games?" $\mathrm{N}=$ novice, $\mathrm{SE}=$ slightly experienced, $\mathrm{E}=$ experienced, $\mathrm{VE}=$ very experienced, and $\mathrm{X}=$ expert.

4 "Which kinds of games do you feel most experienced with?".

TABLE 2: Player scoring and game completion data for Group 1.

\begin{tabular}{lcccc}
\hline & \multicolumn{2}{c}{ Score } & \multicolumn{2}{c}{ Level reached } \\
& Game F & Game R & Game F & Game R \\
\hline P1 & 500 & 250 & 7 & 4 \\
P3 & 150 & 0 & 7 & 3 \\
P5 & 1100 & 250 & End & 7 \\
P7 & 1100 & 700 & End & End \\
\hline
\end{tabular}

TABLE 3: Player scoring and game completion data for Group 2.

\begin{tabular}{lcccc}
\hline & \multicolumn{2}{c}{ Score } & \multicolumn{2}{c}{ Level reached } \\
& Game F & Game R & Game F & Game R \\
\hline P2 & 250 & 200 & 8 & 7 \\
P4 & 1100 & 700 & End & 8 \\
P6 & 1050 & 600 & End & End \\
\hline
\end{tabular}

the path of the orb with the mouse or their finger. This path could be where they wanted the orb to go, how it would move when affected by other operators, or simply where it would go based on the sequence of directions. Players also indicated goal points, by orienting the mouse toward them or pointing at them with a finger, while engaged in forming a plan. Sometimes they would also move an operator to a particular location but keep it in one location instead of placing it and would then trace the path with a finger. In some cases, players did no tracing but placed operators in a way that suggested they had determined a path mentally. Regardless of the number of operators they used or how far ahead they planned, none of the players expressed difficulty (verbally or behaviorally) with tracing a path.

The players in Group 2 also engaged in planning. However, planning in Game $\mathrm{F}$ was different than planning in Game R, due to the exclusion of visualizing the rotation:

"I'm still planning moves ahead but I do not struggle to figure out where I'm going to end up. ... I'm not struggling with rotating in my head....
That's part of the planning but you have to actually do that spatial reasoning to say 'this is going to be here and then it's going to go left' and that's very difficult to make multiple rotations in your mind and then plan in the context of that.... You still have to plan [in Game F] no matter what, there's no way you can randomly complete the game. So you still have to plan but you do not have to put as much effort into planning." (P1)

However, the amount of planning in Game F compared to Game R was potentially greater:

"I know where it's going to be, so I'm not spending my time trying to figure out where it's going to be for the next move. My plan is more from the starting point to the end point; I'm spending time rearranging things so it follows the path. Before [in Game R] I had an end point, I had a beginning, but I had no idea how to go from beginning to end because I couldn't see what each move did. But now I know what each move does, so I'm able to develop a strategy; I can plan more." (P3)

4.2.2. Lower Mental Effort Compared to Game R. People in Group 2 considered Game F to be easier than Game R, primarily due to less mental effort required:

"Well in terms of planning ahead, it's much easier. I can say 'I'm going to go up, I'm going to go right, I'm going to go up, I'm going to go down' I can plan the whole level with very little mental effort, or at least it seems that way." (P1)

"I love this game! I do not have to do the rotations and the ball is the only thing that moves, so right now when I click next the ball is going to move right there. So now I click next, and the ball goes there, and I knew that was going to happen." (P3) 
"This is very different. It's definitely not as complex as the other one. It's still involves some kind of thinking but nowhere near the level of the other one." (P5)

When questioned about the difference in the mental effort between games, the players responded with the following:

"In terms of planning and how hard it is mentally I would say this one is certainly easier. Because I can very easily plan all the moves, I just follow it with the mouse. ...And I can, based on that, place my blocks where I want them. There's no confusion really, saying 'is that really what's going to happen?' It's easier to predict. They're both predictable, if you know what's going to happen, but this is easier much to predict accurately." (P1)

"[the mental effort I need to exert is] significantly reduced, because everything is not moving only one thing is moving in the game. And because only one thing is moving you can keep track of it, you know where it currently is and you know where it's going to end up. And because everything else is not moving, there's only one variable you have to follow. In [Game R] you had to follow many variables, where the door is, where the possible blocks that you're putting are..." (P3)

"The other game was very much mentally draining. With this game it's like, I should be thinking, I can think multiple moves ahead but it's almost like I do not really have to because there's not as much risk involved." (P5)

In elaborating specifically the difference in mental effort, P1 focused on visualizing the rotation as the primary source:

\begin{abstract}
"[Game F] does not require any type of spatial reasoning, mental rotation. That's what's very taxing mentally, especially when it comes to sequential processing of that. This is much easier. You do not really have to do this in your head. I mean, you have to imagine the ball moving but you can literally put your finger there and know where it's going to go. There's very little mental effort." (P1)
\end{abstract}

4.2.3. Low Level of Difficulty. All players in Group 1 indicated that the game was rather easy. They rated the difficulty of the game on the design questionnaire as a 2 or 3 out of 5 (mean $=2.5$ ) and rated the increase in difficulty as a 2 or 3 out of 5 (mean = 2.75). P4 commented that "if I could change the game, I would make it more challenging," while P8 wrote that "I liked that the levels weren't so hard to make me frustrated, but were hard enough for a good challenge." All the players had a chance to see the full progression of difficulty, even if they did not complete the game. When comparing the final level of Game F to the earlier ones, P2 said "it's not really much more difficult" and he indicated that the difficulty was the obstacles that forced resetting the level: "The challenges are, of course, the large number of black holes." All the players indicated that they had no problems or confusion with the UI. P6 even made this more explicit by writing in the design questionnaire that "the controls were concise and could be used effectively."

When comparing the difficulty of Game F and Game R, players in Group 2 indicated that Game F was much easier: "It does not really even compare. The other one [Game R] had just so much more going on that it was way harder. ... I always had to prepare for the next move no matter what, whether or not it allowed me to get closer to the goal." (P5)

All players, in both groups, indicated that the operators gave them more control over the movement of the orb. Since the arrow operators affected the direction of the orb, these were considered the most useful. However, they also made the game much easier, as is mentioned by the players. P4 said "with four arrows you can do anything. It is very easy." P6 explained that the arrows "made the game easier than if we just would have had blocks. It allowed you to control the movements up to 4 moves in advance." However, P8 complained that "the arrows may be too overpowered and those levels [in which arrow operators were available] were generally easier."

4.2.4. Difficulty Affects Depth of Planning. Two of the players in Group 2 said that they could plan up to twelve steps ahead, using all the directions in the movement sequence. The other two said they could only plan about three or four moves ahead. For example, P5 said "[I'm looking] three maybe four [moves ahead]. You always try to look as far as ahead possible, but in the other game it was a lot more difficult to do that. So usually it was just one, maybe two [moves]."

However, the ease with which the players could plan in this game may have discouraged them from planning far in advance. P1 suggested that "It seems I do not really need to plan that much in advance, I can just move. I know where a few moves are going to take me and then just go from there." Giving the player too many arrow operators also seemed to reduce the need to plan further in advance. When asked about the influence of the arrow operators, P7 replied: "They make life easier, for sure. You do not have to construct a long plan, because you can get to each objective every turn. So I found myself thinking less because you do not really have to. It is the same across both games." A similar comment was made by P1, "I'm using the arrows to try to plan multiple moves at once. I like the challenge of planning multiple things and then seeing it all happen, but that's my strategy; the game does not make me do that."

4.2.5. More Trivially Enjoyable Than Game R. Despite how easy the game may have been, the players in Group 1 still enjoyed it. In the design questionnaire, P4 commented " $I$ liked the thinking involved in this game" while P8 commented "I liked how you have to be clever in how to use actions to avoid death." Complaints were directed toward the perceived low difficulty, and not the UI or the mental effort involved.

When players in Group 2 were asked which game they enjoyed playing more, three said that Game $\mathrm{F}$ was more enjoyable because the challenge of the other game was too high for them. One player said that Game $\mathrm{R}$ was more enjoyable because he preferred its higher level of difficulty. 
However, when asked which game they would prefer to play again, two said that they would prefer Game R over Game F and a third one said that he would only play Game $F$ again if it was made harder: "I would get bored playing this all day, as it becomes trivial quickly." (P7) The main difference between the two games was well captured by P5:

\begin{abstract}
"They're both games, but [Game F] is more of a conventional fun game. The other one is more of a mind workout. I see the value of both but depending on the mood I'm in or what I'm prepared to do [I would play one or the other]. I'd be interested in playing both [games] but at different times. If I knew I couldn't play these games outside the context of this [investigation] then I'd ask to play the other one because it would do more for me than playing [Game F]. It is still slightly challenging. If I want a break from work, which is how people normally pick-up games, then I'd pick this one. But, if I wanted something that would really alter my thought process then I'd pick the other one for sure."
\end{abstract}

4.3. Results of Game R. The players of Group 2 played Game $\mathrm{R}$ first, while those in Group 1 played it second. In this game, players reported that the main cognitive task in which they were engaged was visualizing, visualizing required a high amount of mental effort, the high mental effort needed for visualizing also made Game $\mathrm{R}$ more difficult to play than Game F, it was difficult to plan more than a couple moves ahead, and Game $\mathrm{R}$ was considered enjoyable but in a different way than Game F.

4.3.1. Main Cognitive Task: Visualizing. Various comments made by the players while playing the game suggest that they were engaged in planning. For instance, P1 said the following while verbalizing his thoughts:

"Maybe get it into the middle. Let's try this. [placed wall operator] It will go there, and then [clicked action button] and then it's going to fall this way. ...And then it's going to rotate, so if I go like this. [clicked action button] and then, oh that's. . [looked at direction queue, saw next entry was a 180-degree turn] oh boy, I have to plan all these things ahead."

As another example, consider the following excerpt from P3:

"So the ball will end up one rotation here, and this guy [the exit] will end up one rotation here. So what we want is that when the ball gets up here, we want it to fall this way. It will come all the way back down and we want it to go across, so let's take this [wall operator] and place it so that it's here. Now, think about this again....Instead of stopping the ball here let's stop the ball so it's over here, because if the ball stops somewhere over here then it'd be in this quadrant. If I get the ball in the same quadrant [as the goal] then the next time it moves, I can use these four blocks and put them where they need to be."

Similarly, after P5 clicked the action button and did not like the result he said "I should have a put a block here, then I would have got a life after. In this context, you have to look ahead to see where things will be after the rotation."

However, players also visualized the rotation of the play space. As can be seen from the above quotations, visualizing was performed at each step of their plan and thus players could not plan without visualizing. The players engaged in a variety of strategies to help them visualize the rotation of the play space. One tilted his head to see the screen from different orientations. Another arranged the operators on the screen to indicate where certain elements would end up after the rotation. For example, after placing a wall operator at some position on the screen P3 said "this wall is just a placesetter, to mark where the ball will end. I'm using these blocks for other things just so I can visualize what's going on." Most of the players used the mouse or their finger to trace the arc of rotation for some object. Players in Group 1 also performed the same actions, with P2 even explaining his strategy for using the wall operators as mental aids: "I have enough of these [wall operators] that I do not have to think about it, so I'm using the resources as a way to ease the way I think, and then the mental effort will probably decrease."

4.3.2. High Mental Effort Needed for Visualizing. Visualizing had a high mental-resource cost for the players. When P7 was asked what required the most mental effort he responded: "probably visualizing the rotation, then planning to get every coin since I'm committed to that." Similarly, P2 explained that the thing which required the most mental effort was "trying to visualize the effect of each action," and then afterward "I have that sort of visualization in my mind... and I'm trying to put these blocks in that visualization as a component to see what would work." When P5 was asked whether he was thinking about the way in which the play space rotates he replied:

"That's always in the back of my head, because that's the nature of the game. It's a very abstract way of thinking, the whole world moving around, and it's not something that I'm used to in any game in any setting. That's the main problem that I'm having right now."

The high effort can also be seen in the mistakes made by the players and the confusion or frustration expressed upon realizing that the orb moved in a different direction or to a different location than anticipated. For example, after clicking the action button P1 said "that was not how I wanted it to go. ...It rotated the way I expected but I do not know why I thought it was going to fall a different way." Similarly, after placing some operators in anticipation of a certain rotation P3 clicked the action button and, while watching the reaction, said "No! This guy needed to be here!" Even after determining the rotation of the play space, players would frequently double-check the anticipated outcomes to ensure a mistake was not made. This occurred more regularly for some players after they started making mistakes, but all players 
performed double-checking regardless of when they made mistakes.

The players in Group 1 had a similar experience, but it was unexpected given how well they played Game F: "Now I sometimes make mistakes. I thought one thing would happen but now I see that something else happened. But I have to make sure that even if I make mistakes I won't lose." (P4) Likewise, P6 said that the game "is much more frustrating, because you cannot make the ball go where you want it to. [There is] definitely less control." Due to the greater effort involved, some of the Group 1 players said they would just focus on solving the level:

"[in Game F] I would have gone for scoring the coins, or the stars, but in this one the first thing that I am thinking about is getting out of the door. Even though I know that there are only two black holes, but yet because I am not sure about the effects I think that is what I am going to do." (P2)

4.3.3. Higher Difficulty Than Game F. Generally speaking, the players stated that this game was difficult to initially play and took some time to learn. They spent between 5 and 10 minutes on the first two levels of the game, trying to understand how the elements within the play space would rotate and practicing visualizing the rotation. Regardless of the time spent practicing, they all expressed difficulty with visualizing. For example, while verbalizing his thoughts, P3 said: "so it rotates this way, and then the bottom guy becomes this top guy. So, the ball will be here. But that's not where I want it, I want it here. This game is too difficult, [laughs] I have to get through at least level 2!" As another example, while P7 was reflecting on his planning he said "I usually think about where I want to be and then I try to get there, but going more than five [rotations] is really tough. The board flips make it tougher." When talking about the difficulty of predicting the location of the orb, P5 said: "Obviously it's hardest with the inverse flip. Right now the world's gonna invert, and this coin will end up on top and this will end up there, and I cannot do anything to stop that I think. I want the ball to stay here close to the coin, ideally."

On the paper surveys, the players rated the difficulty of the game at a 4 or 5 out of $5($ mean $=4.5)$ and also rated the increase in difficulty at a 3 or 4 out of 5 (mean $=3.5)$. In further elaboration, P5 wrote in the design questionnaire that the game was "very challenging. Not so much a 'fun' game but an exercise for the mind." Similarly, P1 wrote that "the cognitive load and spatial reasoning was demanding. The game was very forgiving in terms of number of moves, which made it seem less difficult than it could have been." The players indicated that the difficulty of the game came from having to visualize the rotation of the play space. For instance, P3 wrote in the design questionnaire that the game was very difficult "because I did not understand what the [180-degree rotation] did or how things rotated. It was hard to visualize the result of a possible action." When asked to elaborate, P3 said:

"The problem is that I cannot see-I'm having a difficult time anticipating where everything is going to be. Because now I know, ok this is here and this is here', and then they will go back to the position where they were before and nothing's changed. But then I do this guy [the 180-degree rotation] and I cannot even make a strategy."

Players in Group 1 indicated that Game R was more difficult than Game F, and that playing Game $\mathrm{R}$ required more mental effort than playing Game F. For instance, P4 mentioned that Game R "is much harder because you cannot predict objects and their place. And if you predict, there will be problems. If I had arrow [operators] in this level it would be so easy." Similarly, P2 explained the difficulty that he had with visualizing:

"First of all, it's definitely hard to understand the way that these blocks and the ball move. It needs more than one or two test actions. Since the effect of the left and right arrows is somehow different than the other ones, this will pose another challenge. So, the first challenge is to understand what are the elements out there doing, in terms of the actions, and then when I could understand that then I could come up with solutions based on using the red blocks."

Finally, P6 was the most explicit about the difference between the two games when he said "this game [Game R] is definitely harder because it's harder to visualize a few moves in advance, especially the flipping. This requires more mental effort [to play]."

4.3.4. Visualizing Limited Planning Depth. Players in both Group 1 and Group 2 were only able to plan a few moves ahead:
"It really is much easier just to compute one move at a time. If there wasn't rotation you could do multiple moves which - It's hard to do that spatial reasoning. To say 'ok, it's going to be like this' and then from there 'it's going to be like this'. I can do it in some cases but then sometimes you make a computation error because you think 'in two stages away it's going to be like this' but then you forgot that something is going to rotate in a certain way." (P1)

P6 mentioned that he was thinking "two moves ahead, maybe three in some cases," while P4 explained that "I just look at the next arrow and I put some things down to avoid losing and be closer to the current goal." When P2 was asked whether he planned a sequence of several actions he replied with:

"No, because the uncertainty of actions and sublevels-a sequence of two or three actions in the same level-are much higher than the previous one [Game F]. The previous one was exact. In this one [Game R], the uncertainty exists and it's to a high degree. So that will stop me thinking ahead of time for maybe even more than one step, because I'm not really good at spatial recognition and memorizing things based on the place that they are or where they are going to be." 
Three of the four players in Group 1 also said that they engaged in more planning in Game F, since it was too difficult in Game $\mathrm{R}$ to create long plans.

4.3.5. Different Enjoyment Than Game F. When asked which game was more enjoyable, three of the four players in Group 1 said Game R was more enjoyable since they preferred the higher level of challenge. For instance, P2 said "I had a more enjoyable time with this game than the previous one," and P6 agreed: "I had more fun playing this one more, because it's harder." P4 disagreed though:

"If I want to say which game I liked more, I liked [Game F] more. This one [Game R] is more challenging, but I think less. It's like you play a game, and it's very hard, but you do not think you just play the hard game."

However, they expressed the opinion that Game R was more about avoiding obstacles. For instance, $\mathrm{P} 8$ said "The previous game required an offensive strategy, whereas this game uses a defensive strategy as you try not to die." When Group 1 players were asked what game they would play again, three said they would prefer to play Game R since Game F was not challenging enough for them:

"It depends if I really wanted to have a challenge or I just wanted to enjoy. If I just wanted to enjoy, then of course the first one [Game F] because I thought I could score more. If I really wanted to head for challenge then this one [Game R]. Solving the challenge in this game is definitely more enjoyable than in the previous game." (P2)

When asked to compare the two games, P2 continued this thought and said: "[These games are] different. The goals are the same, but the way that I am thinking about obtaining those goals are different."

\section{Discussion}

As can be seen from the results, there was a difference between the cognitive gameplay of Game F and Game R. When the players played Game F they engaged in planning, to create a path through the play space, but it seemed that they did not engage in visualizing. Yet, when the same people played Game R they engaged in visualizing the rotation of the play space in a very effortful manner. This suggests that structural differences in interaction may affect cognitive gameplay. However, other implications arise from these results, which can be roughly grouped into three areas: challenge and enjoyment, interaction design, and cognitive gameplay. The implications in these three areas will be discussed in more detail in the subsections below. At the end of this section, we will discuss some of the limitations of this investigation and the conclusions that should not be drawn based on these results.

5.1. Implications for Challenge and Enjoyment. There was a difference in difficulty between Game F and Game R. All the players reported that Game $\mathrm{R}$ was more difficult than Game F, because they needed to visualize the rotation of the play area. In other words, a difference in the cognitive gameplay also resulted in a difference in the difficulty that players experienced while playing the game.

Visualizing the rotation of the play area in Game $\mathrm{R}$ can be considered an additional cognitive challenge that players needed to overcome. By challenge is meant an obstacle which the player must overcome in some way to progress through the game (see [8]). Challenges in a game can be cognitive in nature, such as recognizing a specific series of patterns or working through a logic problem. In the design of cognitive gameplay then, it seems that we can incorporate specific cognitive tasks into the structure of a game by turning them into challenges. From the perspective of systematic design of games, it would be best to introduce cognitive challenges intentionally. If we are not aware of how cognitive tasks can turn into challenges, we may unintentionally disrupt the difficulty balance of our game and potentially turn an enjoyable game into a boring or frustrating one.

Existing research already indicates a correlation between the difficulty of a game and the enjoyment experienced by the player (e.g., $[10,34,35])$. When the player encounters a game with a difficulty level that is too low or too high for their own expertise, they find the game less enjoyable. Such an effect was also seen in this investigation. Over half of the players indicated that they preferred playing Game R over Game F, and this was because they found Game R more difficult and liked that higher degree of difficulty. For them, Game F was enjoyable but too easy for long-term enjoyment. The other players did not prefer playing Game $\mathrm{R}$ and cited the high difficulty as the reason. They found Game $\mathrm{R}$ too difficult, were regularly frustrated, and found relief and enjoyment in Game F. They indicated that Game R was too difficult because they were unable to correctly and efficiently visualize the rotation of the play area. Even though balancing the difficulty of a game should already be a conscious design choice, we need to consider the impact of cognitive gameplay on difficulty since cognitive challenges will affect the difficulty and enjoyment experienced by the player. Similarly, the player may enjoy cognitive challenges; we should then focus on the nature and difficulty of the challenge, since that will probably affect enjoyment more than the presence or absence of cognitive challenges in the game.

Lastly, it is interesting to note that most of the players who preferred playing Game $\mathrm{R}$ were those who played it as the second game. Perhaps the mental effort involved in visualizing rotation may be too much to be immediately introduced. Although the players who played Game R as the second game indicated that they found it harder and enjoyable, they seemed to have less difficulty with visualizing the rotation than the players who encountered it immediately. This could be due to differences in the players' abilities. Another possible explanation is that players who played Game $\mathrm{R}$ second only had to learn how to visualize the rotation. First-time players of Game $\mathrm{R}$ had to learn all the rules of the game while also learning how to visualize the rotation. Since visualizing rotation was so integral to the game, they may not have enough mental resources to learn 
the functionality of various operators as well as discover paths through the level. Thus, it is possible that Game $\mathrm{R}$ required too much mental effort from first-time players. When they played Game F afterwards, they encountered only one new rule (the orb moving in specific directions) and it was much easier to learn. Therefore, introducing rotation in later levels of Game F might be better, as this would give the player the opportunity to first learn the rules of the game and then have more resources available to learn and practice visualizing the rotation.

5.2. Implications for Interaction Design. The difference between Game F and Game R is structural, but it is the reaction component of interaction that was changed. When the action button was clicked in Game F, the reaction was simply that the orb moved in a certain direction. When the action button was clicked in Game $\mathrm{R}$, the reaction was that all objects in the play space rotated in a certain direction. We could attempt to derive conclusions about the relationship between spread of reaction and cognitive gameplay. However, given the nature of this investigation, we should instead merely note that cognitive gameplay may be influenced by changes in the structural elements of interaction. For our investigation, one element of the reaction component was changed. However, an element of the action component could have been changed instead, or a different element of the reaction component could have been changed. Without a framework that identifies possible structural elements of interaction, it will be difficult to comprehensively study the relationship between interaction and cognitive gameplay. This is also true for structural elements other than those related to interaction (e.g., lives, narratives, scoring, and time limits). A framework of structural elements of games and their relationship to cognitive gameplay does not exist yet, though similar frameworks are being developed (e.g., $[14,15])$.

The type of interaction that is available can also influence cognitive gameplay in ways other than those explicitly mentioned by the players in this investigation. For example, the main interaction in Tetris was also used to offload certain cognitive processes [28]. If that interaction was different or could not be used to offload cognition, then the resulting cognitive gameplay would be different; there would be a difference in the distribution of cognitive processes within the player-game cognitive system. A result similar to that found by Kirsh and Maglio [28] was seen in our investigation. When playing Game R, some players used the available operators to help visualize the rotation. This was unintentional in the design of Game R. In general, the player may interact with the game to offload their cognitive processes. However, we can enhance such offloading when we are aware of this possibility and intentionally design the game to better facilitate it. With the example of Game $\mathrm{R}$, we could have allowed the player to mark certain locations in the play space or to watch an animation of the play space rotating in a certain direction. Either of these changes may have assisted players in visualizing. Likewise, we could have designed Game R so that an operator could not be moved once it was placed. This would greatly discourage players from using operators for anything other than their intended purpose and hence would have removed one of their only in-game aids for visualizing.

Finally, the difficulty that players had in visualizing the rotation might have been influenced by the way in which rotation was implemented. Rather than the play space rotating as a continuous whole, only the objects within the play space moved. If players focused on all of the elements and tried to visualize them individually, this may have been why players found rotation difficult to visualize and resource intensive. Additionally, players found the 180-degree rotation the most difficult to understand. This rotation did not involve two 90-degree rotations; instead, objects moved in more-orless a straight line to their destination point. Although objects ended up at a position that was a 180-degree rotation from their starting position, the path these objects took may have confused players. Both points regarding how rotation was implemented suggest that the way in which we implement the design of a computer game may unintentionally affect the difficulty of its tasks. Likewise, it may also affect cognitive gameplay; although this is not something we explored, the method of investigation presented in this paper could be used for such an exploration.

5.3. Implications for Cognitive Gameplay. While many of the implications mentioned in the previous two subsections also apply to cognitive gameplay, two further issues need to be mentioned. First, when looking at the results of this investigation, it is clear that players engaged in planning when playing either Game F or Game R. When playing Game F, players said that they could plan from five to twelve steps ahead. However, most of the time they only planned with the next one or two entries in the direction queue in mind. Most of them reported that they felt the game did not require them to plan further ahead, so they only developed very short plans. They could keep clicking the action button without concern for a more efficient solution, and they had multiple attempts at each level; so if they did not prevent the orb from being destroyed, they could still try the level again. It seems that such few restrictions made Game F rather easy and less cognitively engaging than Game R. Some players tried to challenge themselves by self-enforcing various restrictions, or attempting to achieve a high score, but these activities point to the shallowness of the cognitive gameplay. Hence, even though Game F allowed the players to plan ahead, there were too few in-game restrictions or rewards for encouraging much in the way of long-term planning. Further research, then, is required to explore possibilities for other factors that can be incorporated into a computer game to increase the depth of cognitive gameplay. As previously mentioned, a framework detailing the structural elements of a game and their influence on cognitive gameplay would greatly assist with this.

In Game R, an alternative problem arose. Since planning was dependent on correctly visualizing the rotation of the play space, the players who found it too difficult to visualize the rotation were unable to create any kind of plan. At most, they attempted to guess the result of the first entry in the direction queue and used the operators at their disposal, but they were not able to consider further entries in the queue. 
However, only some of the players found it too difficult, and these players were able to create long plans in Game F. Therefore, it is likely that these players had difficulty planning in Game $\mathrm{R}$ not because of their inability to plan but because of the intense cost of visualizing the rotation. Just as some minor structural change can make the cognitive gameplay too shallow, as in the case of Game F, it could also make the cognitive gameplay too mentally demanding, as in the case of Game R. The resource cost of various tasks, and what factors of interaction can affect this cost, is another possible area of further research.

5.4. Limitations of This Investigation. First and foremost, we did not conduct a study to determine which game produced better or deeper cognitive gameplay. Our interest was purely in exploring the cognitive gameplay that resulted from a single structural change. Although this structural change was associated with the reaction component of interaction, further conceptual clarification is needed in order to study it in more detail. These two games could be studied again, but it is not clear how the same reaction could be translated into other games. Likewise, there are many other structural elements that we could study using this same method, but doing so without an associated conceptual framework will make it difficult to translate the results of such studies into a general framework for designing cognitive gameplay. Existing literature detailing such structural elements tends to be vague in terms of specific components (e.g., [14]), although this could be due to focusing on very general components (e.g., story, challenge) rather than particular ones (e.g., a rule limiting the number of actions). A potential framework for the structural elements of interaction exists (see [16]), but it is not within the context of computer games and may require some adaptation.

In our investigation, we only gathered qualitative data. This was sufficient for exploring the experiences of our players but seems insufficient for conducting a detailed analysis of the differences in cognitive gameplay. In particular, further analysis could be performed on the depth of planning involved, the amount of mental resources used, and the effect of cognitive offloading on any internal visualizing. Interview and in-game questions that were specifically structured for such an analysis would be an improvement over our current investigation. Gathering quantitative data would also be an improvement, particularly for analyzing the memory usage and memory capacity of the players. Other metrics could also be gathered, such as the actions that players performed, the exact time between actions, and the time spent on each level. However, such data requires a much larger sample size for it to be statistically valid, so any future studies would need more participants. The limitation of small sample size prevented quantitative data from being particularly useful, and hence we had to rely on qualitative data. Such limitations are acceptable for the investigation presented in this paper, but they need to be overcome for more formal studies in the future.

\section{Conclusions}

This paper presented an exploratory investigation of the effect of structural variations of interaction on cognitive gameplay.
Two computer games were designed and developed (Game $\mathrm{F}$ and Game R). These games were isomorphic at a deep structural level, and their only difference was in one component of interaction. Eight volunteers played both of these games, their behavior and comments were recorded, and a difference in cognitive gameplay was observed. When they played Game $\mathrm{F}$ they were primarily engaged in planning, but when they played Game R they were engaged in visualizing rotation. The mental load needed to visualize was reported as being so heavy that the players found it difficult or impossible to also engage in planning. Also, there was a difference in difficulty between the two games, which led to a difference in enjoyment. All the players said that Game R was the harder of the two. Those who found Game R too difficult preferred Game F, while the rest found Game F too easy and preferred to play Game R.

This investigation was conducted to better understand how we can study the relationship between structural components of interaction and cognitive gameplay. The design process that we used in this paper (see [20]) can enable researchers to develop numerous isomorphic computer games. Since we can control the structural differences between these games, we can also control the features that we want to isolate for further study. This process has not been used to study the effects of structural elements until this paper. Even though we only explored one structural element of interaction, the investigation procedure presented in this paper could be used to study many different interaction elements and other structural elements unrelated to interaction. As previously mentioned though, a framework identifying and conceptualizing these elements would greatly assist in such an investigation.

Having control over the differences between games allows us to draw stronger conclusions about how to design cognitive gameplay. We only investigated the cognitive processes in which the players were engaged and not whether the game was responsible for any improvement in the players. However, more detailed and elaborate studies need to be conducted, building upon the simple investigation presented in this paper, to provide evidence for the microlevel features of a computer game that improve or hinder specific kinds and depth of cognitive gameplay. In all such cases, more formal studies of the relationship between the structure of interaction and cognitive gameplay need to be conducted, and a more systematic conceptual framework of the structure of interaction in games needs to be developed. It is our hope that such studies will be conducted in the future, and that this paper provides a promising start.

\section{Conflict of Interests}

The authors declare that there is no conflict of interests regarding the publication of this paper.

\section{References}

[1] S. Deterding, M. Sicart, L. Nacke, K. O'Hara, and D. Dixon, "Gamification: using game design elements in non-gaming contexts," in Proceedings of the Annual Conference Extended 
Abstracts on Human Factors in Computing Systems (CHI EA '11), pp. 2425-2428, 2011.

[2] M. P. J. Habgood and S. E. Ainsworth, "Motivating children to learn effectively: exploring the value of intrinsic integration in educational games," Journal of the Learning Sciences, vol. 20, no. 2, pp. 169-206, 2011.

[3] H. A. Spires, "21st century skills and serious games: preparing the N generation," in Serious Educational Games: From Theory to Practice, L. A. Annetta, Ed., pp. 13-23, Sense Publishers, Rotterdam, The Netherlands, 2008.

[4] L. Ermi and F. Mäyrä, "Fundamental components of the gameplay experience: analysing immersion," in Proceedings of the 2nd International Conference on Digital Games Research Association (DiGRA '05), 2005.

[5] K. Salen and E. Zimmerman, Rules of Play: Game Design Fundamentals, vol. 37, MIT Press, Cambridge,UK, 2004.

[6] K. Sedig, "From play to thoughtful learning: a design strategy to engage children with mathematical representations," Journal of Computers in Mathematics and Science Teaching, vol. 27, no. 1, pp. 65-101, 2008.

[7] J. Elliott, L. Adams, and A. Bruckman, "No magic bullet: 3D video games in education," in Proceedings of the 5th International Conference of the Learning Sciences (ICLS '02), pp. 23-26, Seattle, Wash, USA, 2002.

[8] E. Adams, Fundamentals of Game Design, Pearson Education, Berkeley, Calif, USA, 2nd edition, 2010.

[9] R. Haworth, P. Parsons, and K. Sedig, "Design of digital cognitive games: some considerations," Cognitive Technology Journal, vol. 18, no. 1, pp. 22-27, 2013.

[10] J. T. Alexander, J. Sear, and A. Oikonomou, "An investigation of the effects of game difficulty on player enjoyment," Entertainment Computing, vol. 4, no. 1, pp. 53-62, 2013.

[11] T. M. Connolly, E. A. Boyle, E. MacArthur, T. Hainey, and J. M. Boyle, "A systematic literature review of empirical evidence on computer games and serious games," Computers and Education, vol. 59, no. 2, pp. 661-686, 2012.

[12] K. Isbister and N. Schaffer, Game Usability, Morgan Kaufmann, Burlington, Mass, USA, 2008.

[13] P. Sweetser and P. Wyeth, "GameFlow: a model for evaluating player enjoyment in games," Computers in Entertainment, vol. 3, no. 3, pp. 1-24, 2005.

[14] V. Aleven, E. Myers, M. Easterday, and A. Ogan, "Toward a framework for the analysis and design of educational games," in Proceedings of the 3rd IEEE International Conference on Digital Game and Intelligent Toy Enhanced Learning (DIGITEL '10), pp. 69-76, IEEE, Kaohsiung, Taiwan, April 2010.

[15] W. L. Bedwell, D. Pavlas, K. Heyne, E. H. Lazzara, and E. Salas, "Toward a taxonomy linking game attributes to learning: an empirical study," Simulation \& Gaming, vol. 43, no. 6, pp. 729760, 2012.

[16] K. Sedig, P. Parsons, M. Dittmer, and R. Haworth, "Humancentered interactivity of visualization tools: micro- and macrolevel considerations," in Handbook of Human Centric Visualization, W. Huang, Ed., pp. 717-743, Springer, New York, NY, USA, 2014.

[17] K. L. Powers, P. J. Brooks, N. J. Aldrich, M. A. Palladino, and L. Alfieri, "Effects of video-game play on information processing: a meta-analytic investigation," Psychonomic Bulletin and Review, vol. 20, no. 6, pp. 1055-1079, 2013.

[18] M. Á. Quiroga, F. J. Román, A. Catalán et al., "Videogame performance (not always) requires intelligence," International
Journal of Online Pedagogy and Course Design, vol. 1, no. 3, pp. 18-32, 2011.

[19] K. Sedig, M. Klawe, and M. Westrom, "Role of interface manipulation style and scaffolding on cognition and concept learning in learnware," ACM Transactions on Computer-Human Interaction, vol. 8, no. 1, pp. 34-59, 2001.

[20] K. Sedig and R. Haworth, "Creative design of digital cognitive games: application of cognitive toys and isomorphism," Bulletin of Science, Technology \& Society, vol. 32, no. 5, pp. 413-426, 2012.

[21] J. S. Brown, A. Collins, and P. Duguid, "Situated cognition and the culture of learning," Educational Researcher, vol. 18, no. 1, pp. 32-42, 1989.

[22] J. Hollan, E. Hutchins, and D. Kirsh, "Distributed cognition: toward a new foundation for human-computer interaction research," ACM Transactions on Computer-Human Interaction, vol. 7, no. 2, pp. 174-196, 2000.

[23] G. Lakoff and M. Johnson, Philosophy in The Flesh: The Embodied Mind and Its Challenge to Western Thought, Basic Books, New York, NY, USA, 1999.

[24] G. Salomon, Ed., Distributed Cognitions: Psychological and Educational Considerations, Cambridge University Press, Cambridge, UK, 1993.

[25] A. Clark, Supersizing the Mind, Oxford University Press, Oxford, UK, 2008.

[26] E. Hutchins, Cognition in the Wild, MIT Press, 1995.

[27] P. Parsons and K. Sedig, "Distribution of information processing while performing complex cognitive activities with visualization tools," in Handbook of Human Centric Visualization, pp. 693-715, Springer, New York, NY, USA, 2014.

[28] D. Kirsh and P. Maglio, "On distinguishing epistemic from pragmatic action," Cognitive Science, vol. 18, no. 4, pp. 513-549, 1994.

[29] D. Kirsh, "Metacognition, distributed cognition and visual design," in Cognition, Education, and Communication Technology, pp. 1-22, 2005.

[30] G. Salomon and D. Perkins, "Do technologies make us smarter? Intellectual amplification with, of, and through technology," in Intelligence and Technology: The Impact of Tools on the Nature and Development of Human Abilities, R. Sternberg and D. D. Preiss, Eds., pp. 71-86, Lawrence Erlbaum, Mahwah, NJ, USA, 2005.

[31] E. Aarseth, "Playing research: methodological approaches to game," in 5th International Digital Arts and Culture Conference, B. K. Walther, Ed., Fine Art Forum, 2003.

[32] W. Dou, C. Ziemkiewicz, L. Harrison et al., "Comparing different levels of interaction constraints for deriving visual problem isomorphs," in Proceedings of the IEEE Symposium on Visual Analytics Science and Technology (VAST '10), pp. 195-202, Piscataway, NJ, USA, October 2010.

[33] J. Cohen, “A power primer," Psychological Bulletin, vol. 112, no. 1, pp. 155-159, 1992.

[34] B. Cowley, D. Charles, M. Black, and R. Hickey, "Toward an understanding of flow in video games," Computers in Entertainment, vol. 6, article 20, 2008.

[35] K. Sedig, "Toward operationalization of "flow" in mathematics learnware," Computers in Human Behavior, vol. 23, no. 4, pp. 2064-2092, 2007. 

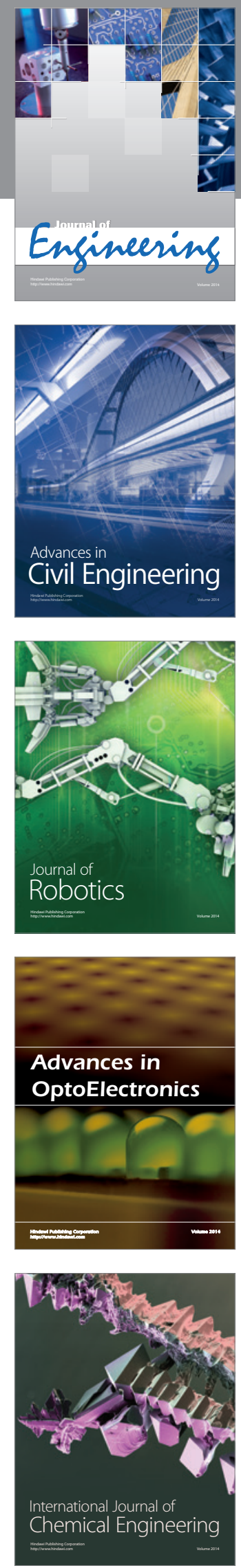

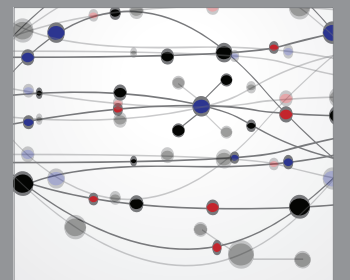

The Scientific World Journal
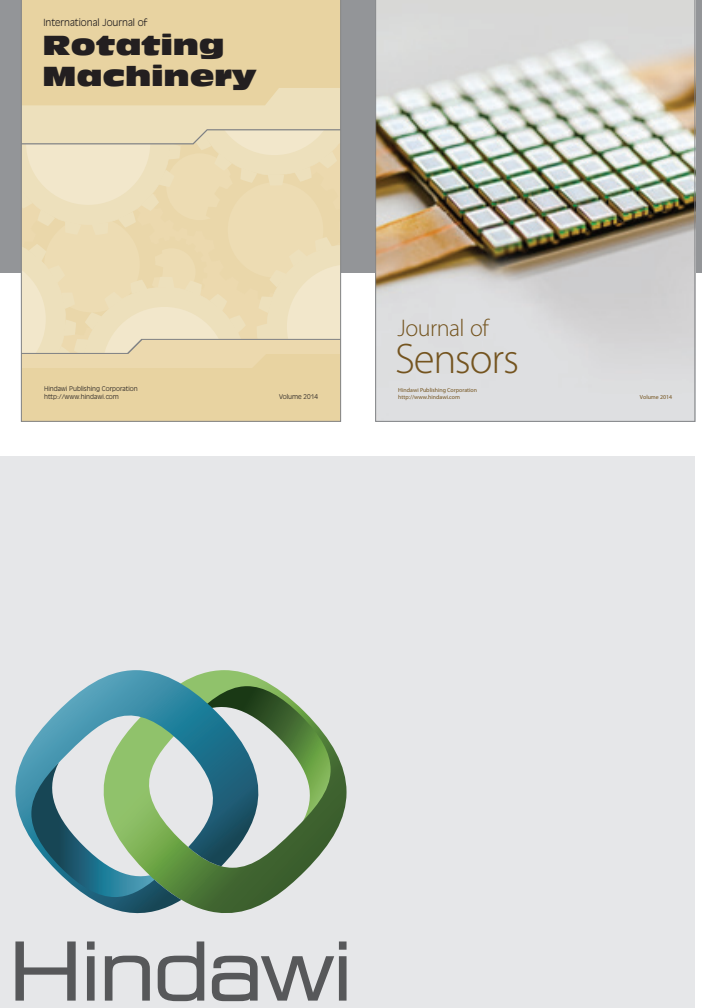

Submit your manuscripts at http://www.hindawi.com
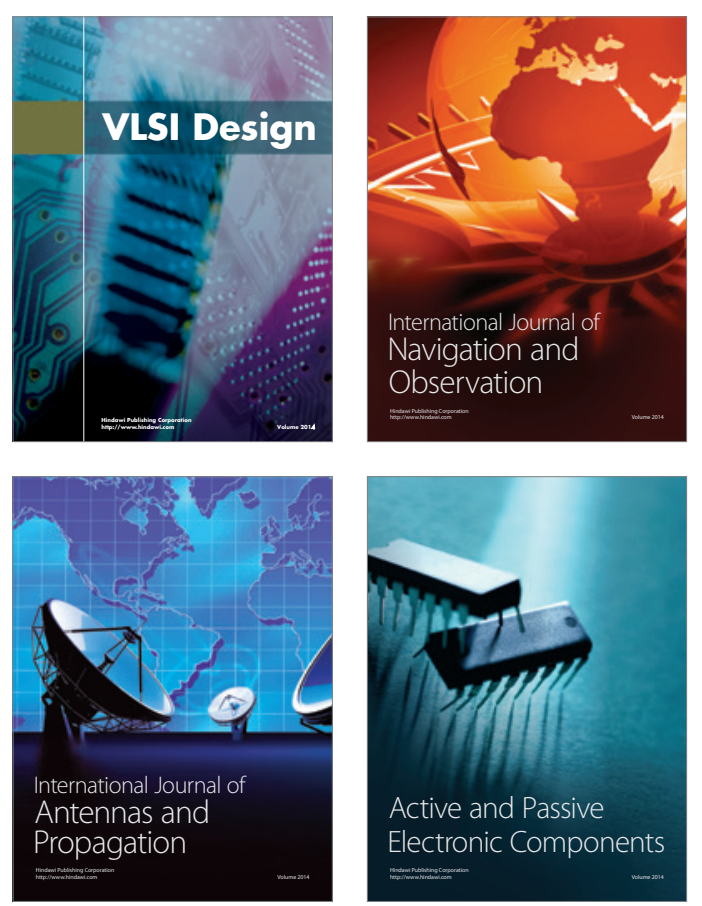
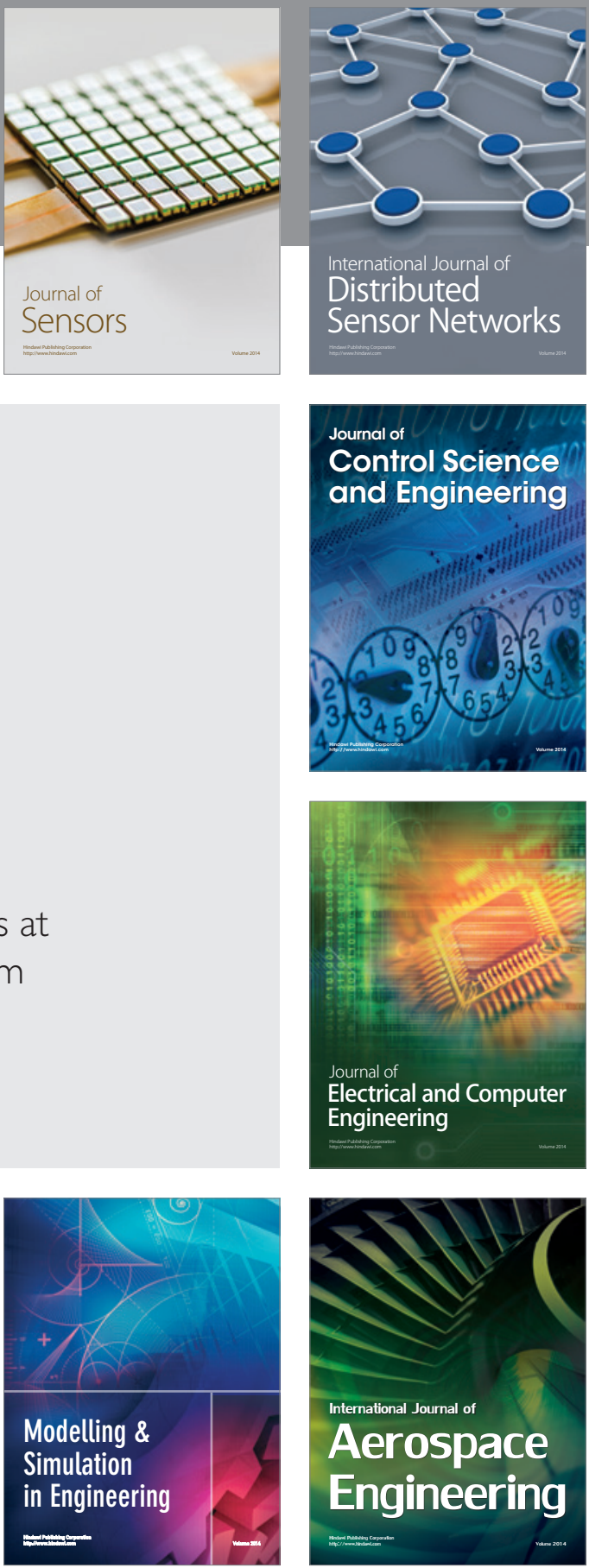

Journal of

Control Science

and Engineering
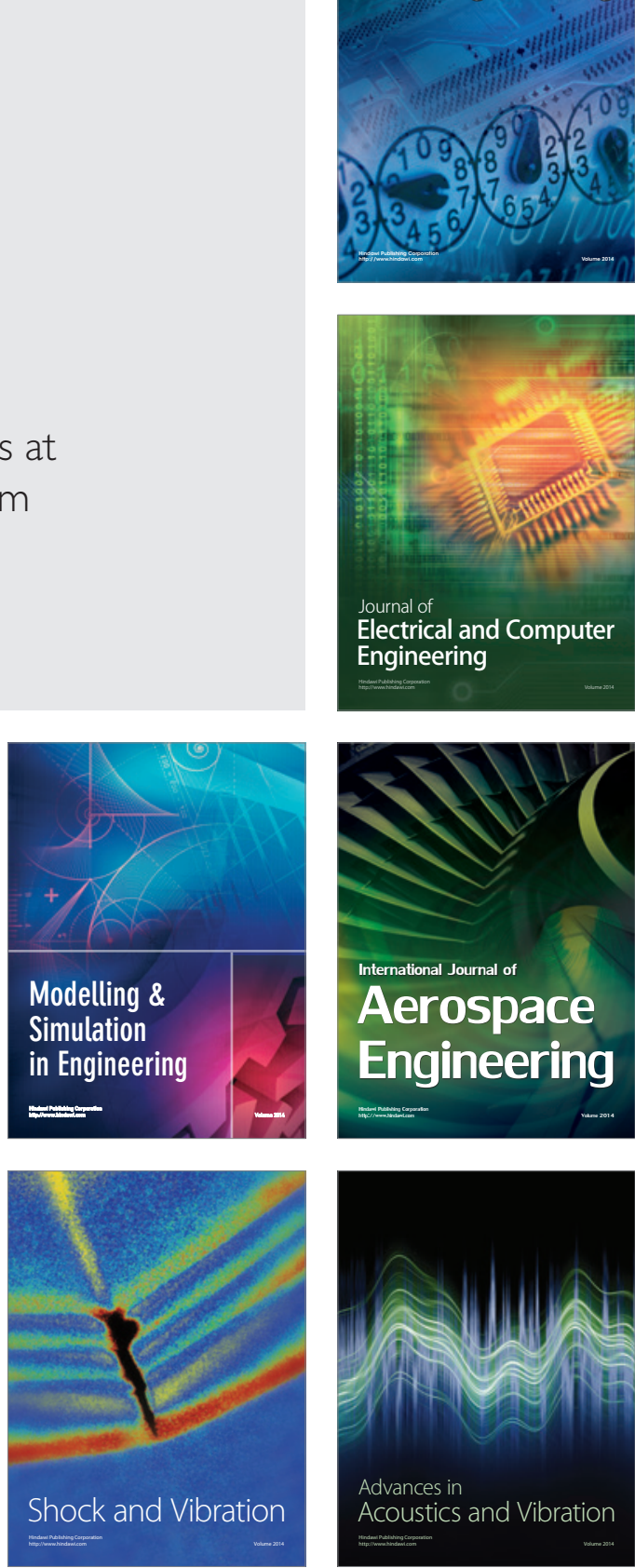